\title{
Analysis of multiple factors involved in acute progressive cerebral infarction and extra- and intracranial arterial lesions
}

\author{
YUEFU CHEN ${ }^{1}$, YAJIE LIU ${ }^{2}$, CHENGHONG LUO ${ }^{1}$, WEIHENG LU ${ }^{1}$ and BINRU SU ${ }^{1}$ \\ ${ }^{1}$ Department of Neurology, Shilong People's Hospital, Southern Medical University, Dongguan, Guangdong 523326; \\ ${ }^{2}$ Department of Neurology, Zhujiang Hospital, Southern Medical University, Guangzhou, Guangdong 510282, P.R. China
}

Received November 4, 2013; Accepted March 3, 2014

DOI: $10.3892 /$ etm.2014.1624

\begin{abstract}
In order to identify the potential factors involved in the development of acute progressive cerebral infarction (PCI), the association between potential risk factors and extraand intracranial arterial lesions was investigated. A total of 608 patients underwent cerebral angiography to analyze the morphological characteristics between the PCI and NPCI groups. In addition, data from numerous cases of extra- and intracranial arterial lesions were collected and compared with the control groups, and the associations between the severity of arterial lesions and the potential influential factors were analyzed. In the blood vessels responsible for cerebral infarction, various degrees of atherosclerotic plaques and stenosis were observed. Age, high-density lipoprotein (HDL) levels, glycosylated hemoglobin and blood pressure affected the degrees of hardening, plaques and stenosis. Analysis of cerebral artery stenosis revealed that age, diabetes mellitus and plasma fibrinogen were risk factors for cerebral artery stenosis, while the HDL/low density lipoprotein ratio was a protective factor. Therefore, the results of the present study indicate that the lesions of blood vessels are a major pathological change in PCI and multiple factors are involved in the pathogenesis.
\end{abstract}

\section{Introduction}

Progressive cerebral infarction (PCI) is a brain disorder caused by insufficient blood supply. Cerebral infarction may lead to cerebral ischemia, hypoxia, necrosis and finally neurological deficit (1). Recently, studies of cerebral infarction have focused on identifying risk factors. Multiple measures have also been taken, including health education, acute-stage patient care, vascular stenting and surgery, neuroradiology,

Correspondence to: Dr Binru Su, Department of Neurology, Shilong People's Hospital, Southern Medical University, Dongguan, Guangdong 523326, P.R. China

E-mail: subinru@gmail.com

Key words: progressive cerebral infarction, carotid atherosclerosis, cerebral artery stenosis, stroke in progression early rehabilitation. However, the prevention and treatment of cerebral infarction remains largely unsuccessful and the prognosis is severe. Between 50 and $70 \%$ of surviving patients are left with paralysis, aphasia and dementia (2). Therefore, it is particularly important to identify risk factors for the development of PCI.

At present, there are no ideal strategies that effectively prevent the progression of cerebral infarction. With the rapid progress in the treatment of intravascular hydrocephalus and the continuous improvement of interventional equipment, stenting and angioplasty are feasible in the treatment of intracranial vascular stenosis (3). These treatments are recommended for patients with intracranial arterial stenosis who do not respond well to medical treatment or whose arterial stenosis is $>50 \%$, according to the guidelines from the American Society of Interventional and Therapeutic Neuroradiology, the American Society of Interventional Radiology and the American Society of Neuroradiology (4). However, the clinical value of vascular balloon angioplasty and stent implantation in preventing the progression of cerebral infarction remains unknown.

The present study enrolled patients with PCI and those with non-progressive cerebral infarction (NPCI) in order to compare and analyze the cerebral angiographic characteristics. Differences in vascular stenosis and vascular morphology were revealed by cerebral angiography. The aim of the present study was to provide further theoretical basis for interventional therapy of cerebrovascular disease.

\section{Materials and methods}

Case selection criteria. Data from 608 PCI patients (male 419, female 189) admitted to the Department of Internal Medicine at Shilong People's Hospital (Guangdong, China) were collected between May 2010 and May 2013. The inclusion criteria met the diagnostic criteria set in the first edition of the Chinese Guidelines for Cerebrovascular Disease Prevention (5) and were confirmed by head computed tomography or magnetic resonance imaging examinations. The patients were divided into two groups: PCI and NPCI groups. The PCI group included patients who had been admitted within $24 \mathrm{~h}$ after the onset of the disease, but had not been treated within $6 \mathrm{~h}$ of onset. The diseases were progressing and the patients scored $\geq 2$ points according to the United 
Table I. Association of potential risk factors with severity of carotid atherosclerosis.

\begin{tabular}{|c|c|c|c|c|c|c|c|}
\hline \multirow[b]{2}{*}{ Risk factor } & \multicolumn{4}{|c|}{ Carotid atherosclerosis } & \multirow[b]{2}{*}{ Total } & \multirow{2}{*}{$\begin{array}{c}\text { Test } \\
\text { statistic }\end{array}$} & \multirow[b]{2}{*}{ P-value } \\
\hline & Normal & Hardening & Plaque & Stenosis & & & \\
\hline \multicolumn{8}{|l|}{ Gender, n $(\%)$} \\
\hline Male & $60(69.0)$ & $67(67.7)$ & 197 (69.6) & $62(74.7)$ & $386(69.9)$ & 1.189 & 0.756 \\
\hline Female & $27(31.0)$ & $32(32.3)$ & $86(30.4)$ & $21(25.3)$ & $166(30.1)$ & & \\
\hline \multicolumn{8}{|l|}{ Diabetes, n (\%) } \\
\hline No & $75(86.2)$ & $75(75.8)$ & $185(65.4)$ & $50(60.2)$ & $385(69.7)$ & 18.988 & $<0.01$ \\
\hline Yes & $12(13.8)$ & $24(24.2)$ & $98(34.6)$ & $33(39.8)$ & $167(30.3)$ & & \\
\hline \multicolumn{8}{|l|}{ Hypertension, n (\%) } \\
\hline No & $49(56.3)$ & $24(24.2)$ & $37(13.1)$ & $8(9.6)$ & $118(21.4)$ & 82.107 & $<0.01$ \\
\hline Yes & $38(43.7)$ & $75(75.8)$ & $246(86.9)$ & $75(90.4)$ & 434 (78.6) & & \\
\hline \multicolumn{8}{|l|}{ Hyperlipidemia, n (\%) } \\
\hline No & $56(64.4)$ & $51(51.5)$ & $127(45.0)$ & $39(47.0)$ & $273(49.5)$ & 10.312 & 0.016 \\
\hline Yes & $31(35.6)$ & $48(48.5)$ & $155(55.0)$ & $44(53.0)$ & $278(50.5)$ & & \\
\hline \multicolumn{8}{|l|}{ Smoking, n (\%) } \\
\hline No & $60(69.0)$ & $58(58.6)$ & $172(60.8)$ & $43(51.8)$ & $333(60.3)$ & 5.379 & 0.146 \\
\hline Yes & $27(31.0)$ & $41(41.4)$ & $111(39.2)$ & $40(48.2)$ & $219(39.7)$ & & \\
\hline \multicolumn{8}{|l|}{ Wine consumption, $\mathrm{n}(\%)$} \\
\hline No & $78(89.7)$ & $87(87.9)$ & $241(85.2)$ & $71(85.5)$ & $477(86.4)$ & 1.393 & 0.707 \\
\hline Yes & $9(10.3)$ & $12(12.1)$ & $42(14.8)$ & $12(14.5)$ & $75(13.6)$ & & \\
\hline Age, years ${ }^{\mathrm{a}}$ & $53.2 \pm 11.6$ & $62.3 \pm 11.3$ & $66.4 \pm 10.0$ & $68.6 \pm 8.3$ & & 43.4 & $<0.01$ \\
\hline $\begin{array}{l}\text { Systolic blood } \\
\text { pressure, } \mathrm{mmHg}^{\mathrm{a}}\end{array}$ & $136.3 \pm 21.3$ & $151.3 \pm 23.8$ & $153.2 \pm 23.5$ & $153.6 \pm 22.1$ & & 12.9 & $<0.01$ \\
\hline $\begin{array}{l}\text { Diastolic blood } \\
\text { pressure, } \mathrm{mmHg}^{\mathrm{a}}\end{array}$ & $81.2 \pm 12.8$ & $85.3 \pm 13.3$ & $85.2 \pm 12.3$ & $83.7 \pm 9.1$ & & 2.6 & 0.049 \\
\hline Platelet count $\left(10^{9} / 1\right)^{\mathrm{a}}$ & $235.788 \pm 67.761$ & $253.153 \pm 95.158$ & $237.735 \pm 76.974$ & $236.602 \pm 70.103$ & & 1.135 & 0.334 \\
\hline International normalized ratio & $1.001 \pm 0.080$ & $1.006 \pm 0.088$ & $1.033 \pm 0.109$ & $1.041 \pm 0.170$ & & 2.911 & 0.034 \\
\hline Plasma fibrinogen $(g / l)^{a}$ & $3.301 \pm 0.789$ & $3.564 \pm 0.796$ & $3.805 \pm 0.990$ & $3.862 \pm 1.011$ & & 7.297 & $<0.01$ \\
\hline Total cholesterol $(\mathrm{mmol} / \mathrm{l})^{\mathrm{a}}$ & $4.675 \pm 1.208$ & $4.959 \pm 1.137$ & $4.980 \pm 1.156$ & $5.043 \pm 1.499$ & & 1.639 & 0.179 \\
\hline Triglyceride $(\mathrm{mmol} / \mathrm{l})^{\mathrm{a}}$ & $1.661 \pm 1.165$ & $1.741 \pm 1.112$ & $1.738 \pm 1.069$ & $1.643 \pm 0.937$ & & 0.254 & 0.859 \\
\hline $\operatorname{HDL}(\mathrm{mmol} / \mathrm{l})^{\mathrm{a}}$ & $1.087 \pm 0.334$ & $1.226 \pm 0.508$ & $1.135 \pm 0.549$ & $1.114 \pm 0.340$ & & 1.451 & 0.227 \\
\hline $\mathrm{LDL}(\mathrm{mmol} / \mathrm{l})^{\mathrm{a}}$ & $2.596 \pm 0.992$ & $2.905 \pm 0.993$ & $2.773 \pm 1.002$ & $2.936 \pm 1.123$ & & 2.039 & 0.107 \\
\hline $\mathrm{HLR}^{\mathrm{a}}$ & $0.480 \pm 0.253$ & $0.492 \pm 0.405$ & $0.478 \pm 0.330$ & $0.451 \pm 0.397$ & & 0.219 & 0.883 \\
\hline \multicolumn{8}{|l|}{ Fasting blood glucose } \\
\hline$(\mathrm{mmol} / \mathrm{l})^{\mathrm{a}}$ & $5.878 \pm 1.691$ & $6.222 \pm 2.006$ & $6.698 \pm 3.055$ & $6.866 \pm 3.622$ & & 2.640 & 0.049 \\
\hline Glycated hemoglobin $(\%)^{\mathrm{a}}$ & $6.233 \pm 1.948$ & $6.197 \pm 1.415$ & $6.942 \pm 2.016$ & $6.970 \pm 1.881$ & & 4.122 & 0.007 \\
\hline Homocysteine $(\mu \mathrm{mol} / \mathrm{l})^{\mathrm{a}}$ & $13.574 \pm 5.586$ & $13.941 \pm 4.898$ & $14.849 \pm 6.470$ & $15.002 \pm 5.258$ & & 1.031 & 0.379 \\
\hline
\end{tabular}

${ }^{a}$ Mean \pm SD. HDL, high-density lipoprotein; LDL, low-density lipoprotein; HLR, HDL/LDL ratio.

States National Institute of Health Stroke Scale (NIHSS) (6). The NPCI group (control group) included patients who were admitted within $24 \mathrm{~h}$ after onset and whose diseases had reached the peak, thus progression had stopped $6 \mathrm{~h}$ after onset. These patients scored $<2$ points on the NIHSS. The study protocol was approved by the Institutional Ethical Committee for Research on Human Subjects (Guangzhou, China) and informed written consent was obtained from each patient.

Carotid artery ultrasonography. To examine the extent of common carotid artery atherosclerosis, the intima media thickness (IMT) and the vessel diameters were measured by ultrasonography. The severity of the carotid artery lesions was classified into four groups: Normal (IMT $\leq 0.9 \mathrm{~mm}$ ), hardening $(0.9 \mathrm{~mm}<\mathrm{IMT} \leq 1.5 \mathrm{~mm})$, plaque formation (IMT>1.5 $\mathrm{mm}$ ) and stenosis (narrowing, $>30 \%$ ).

Cerebral angiography. A Seldinger puncture was created in the femoral arteries of patients from the two groups. Angiography was performed using catheters at the aortic arch, bilateral common carotid arteries and vertebral arteries. Based on the North America Symptomatic Carotid Endarterectomy Trial method (7), vascular stenosis was 
Table II. Association between potential risk factors with the severity of cerebral artery stenosis.

\begin{tabular}{|c|c|c|c|c|c|}
\hline \multirow[b]{2}{*}{ Risk factor } & \multicolumn{2}{|c|}{ Cerebral artery stenosis } & \multirow[b]{2}{*}{ Total } & \multirow[b]{2}{*}{ Test statistic } & \multirow[b]{2}{*}{ P-value } \\
\hline & Narrowing $\leq 50 \%$ & Narrowing $>50 \%$ & & & \\
\hline \multicolumn{6}{|l|}{ Gender, n (\%) } \\
\hline Male & $185(68.0)$ & $234(69.6)$ & $419(68.9)$ & 0.186 & 0.666 \\
\hline Female & $87(32.0)$ & $102(30.4)$ & $189(31.1)$ & & \\
\hline \multicolumn{6}{|l|}{ Diabetes, n (\%) } \\
\hline No & $221(81.3)$ & $209(62.2)$ & $430(70.7)$ & 26.339 & $<0.001$ \\
\hline Yes & $51(18.8)$ & $127(37.8)$ & $178(29.3)$ & & \\
\hline \multicolumn{6}{|l|}{ Hypertension, n (\%) } \\
\hline No & $79(29.0)$ & $50(14.9)$ & $129(21.2)$ & 18.039 & $<0.001$ \\
\hline Yes & $193(71.0)$ & $286(85.1)$ & $479(78.8)$ & & \\
\hline \multicolumn{6}{|l|}{ Hyperlipidemia, n (\%) } \\
\hline No & $154(56.6)$ & $149(44.5)$ & $303(49.9)$ & 8.850 & 0.003 \\
\hline Yes & $118(43.4)$ & $186(55.5)$ & $304(50.1)$ & & \\
\hline \multicolumn{6}{|l|}{ Smoking, n (\%) } \\
\hline No & $185(68.0)$ & $195(58.0)$ & $380(62.5)$ & 6.387 & 0.011 \\
\hline Yes & $87(32.0)$ & $141(42.0)$ & $228(37.5)$ & & \\
\hline \multicolumn{6}{|l|}{ Wine consumption, $\mathrm{n}(\%)$} \\
\hline No & $246(90.4)$ & $281(83.6)$ & $527(86.7)$ & 6.037 & 0.014 \\
\hline Yes & $26(9.6)$ & $55(16.4)$ & $81(13.3)$ & & \\
\hline Age, years ${ }^{a}$ & $60.7 \pm 12.1$ & $65.8 \pm 10.4$ & & -5.6 & $<0.01$ \\
\hline Systolic blood pressure, $\mathrm{mmHg}^{\mathrm{a}}$ & $147.9 \pm 24.9$ & $151.4 \pm 22.7$ & & -1.8 & 0.065 \\
\hline Diastolic blood pressure, $\mathrm{mmHg}^{\mathrm{a}}$ & $84.0 \pm 12.7$ & $84.0 \pm 12.0$ & & 0.026 & 0.979 \\
\hline Platelet count $\left(10^{9} / 1\right)^{\mathrm{a}}$ & $237.345 \pm 78.032$ & $245.263 \pm 78.838$ & & -1.229 & 0.220 \\
\hline International normalized ratio ${ }^{a}$ & $1.029 \pm 0.101$ & $1.020 \pm 0.118$ & & 0.884 & 0.377 \\
\hline Plasma fibrinogen $(\mathrm{g} / \mathrm{l})^{\mathrm{a}}$ & $3.460 \pm 0.859$ & $3.871 \pm 1.045$ & & -5.124 & $<0.001$ \\
\hline Total cholesterol $(\mathrm{mmol} / \mathrm{l})^{\mathrm{a}}$ & $4.862 \pm 1.199$ & $5.023 \pm 1.206$ & & -1.632 & 0.103 \\
\hline Triglyceride $(\mathrm{mmol} / \mathrm{l})^{\mathrm{a}}$ & $1.642 \pm 0.996$ & $1.753 \pm 1.140$ & & -1.27 & 0.204 \\
\hline $\mathrm{HDL}(\mathrm{mmol} / \mathrm{l})^{\mathrm{a}}$ & $1.191 \pm 0.447$ & $1.116 \pm 0.491$ & & 1.942 & 0.053 \\
\hline $\mathrm{LDL}(\mathrm{mmol} / \mathrm{l})^{\mathrm{a}}$ & $2.715 \pm 1.011$ & $2.846 \pm 0.990$ & & -1.598 & 0.111 \\
\hline $\mathrm{HLR}^{\mathrm{a}}$ & $0.520 \pm 0.408$ & $0.444 \pm 0.252$ & & 2.686 & 0.008 \\
\hline Fasting blood glucose $(\mathrm{mmol} / \mathrm{l})^{\mathrm{a}}$ & $6.000 \pm 2.149$ & $6.846 \pm 3.188$ & & -3.874 & $<0.001$ \\
\hline Glycated hemoglobin $(\%)^{\mathrm{a}}$ & $6.415 \pm 1.837$ & $7.009 \pm 1.985$ & & -3.115 & 0.002 \\
\hline Homocysteine $(\mu \mathrm{mol} / \mathrm{l})^{\mathrm{a}}$ & $14.084 \pm 4.669$ & $14.992 \pm 6.561$ & & -1.615 & 0.107 \\
\hline
\end{tabular}

${ }^{a}$ Mean \pm SD. HDL, high-density lipoprotein; LDL, low-density lipoprotein; HLR, HDL/LDL ratio.

assessed by doctors experienced in neurointervention. The degree of cerebral artery stenosis was classified into two groups based on the reduction in vessel diameter: Mild stenosis $(\leq 50 \%)$ and severe stenosis $(>50 \%)$. The severity of arterial lesions was evaluated.

Statistical analysis. Continuous data are presented as mean \pm SD and were analyzed with a Student's t-test or one-way analysis of variance (when the variance was irregular Welch correction was used). Categorical data were analyzed with a $\chi^{2}$ test. To identify the potential risk factors for the development of PCI, the linear regression method and multivariate logistic regression analyses were used. $\mathrm{P}<0.05$ was considered to indicate a statistically significant difference.

\section{Results}

Single factor analysis. Associations between multiple potential risk factors and carotid artery atherosclerosis were firstly analyzed. As shown in Table I, the incidence rate of diabetes was significantly higher in patients with carotid artery atherosclerosis when compared with those with normal carotid arteries $\left(\chi^{2}=18.988 ; \mathrm{P}<0.01\right)$. As the severity of atherosclerosis increased, the diabetes incidence also increased, indicating the involvement of diabetes in the pathogenesis of carotid artery atherosclerosis. Similarly to diabetes, the incidence of hypertension was also significantly higher in patients with carotid artery atherosclerosis when compared with those with normal carotid arteries $\left(\chi^{2}=82.107 ; \mathrm{P}<0.01\right)$. The incidence of hypertension increased to $90 \%$ in patients with carotid artery stenosis, demonstrating the 
Table III. Association between potential risk factors and the severity of stroke.

\begin{tabular}{|c|c|c|c|c|c|c|}
\hline Risk factor & No stroke & $\begin{array}{c}\text { Stroke with } \\
\text { large artery } \\
\text { atherosclerosis }\end{array}$ & $\begin{array}{l}\text { Stroke with } \\
\text { small artery } \\
\text { atherosclerosis }\end{array}$ & Total & Test statistic & P-value \\
\hline \multicolumn{7}{|l|}{ Gender, n (\%) } \\
\hline Male & $113(64.6)$ & $154(70.0)$ & 109 (73.6) & $376(69.2)$ & \multirow[t]{2}{*}{3.201} & \multirow[t]{2}{*}{0.202} \\
\hline Female & $62(35.4)$ & $66(30.0)$ & $39(26.4)$ & $167(30.8)$ & & \\
\hline \multicolumn{7}{|l|}{ Diabetes, n (\%) } \\
\hline No & $140(80.0)$ & $131(59.5)$ & 109 (73.6) & $380(70.0)$ & \multirow[t]{2}{*}{20.714} & \multirow[t]{2}{*}{$<0.001$} \\
\hline Yes & $35(20.0)$ & $89(40.5)$ & $39(26.4)$ & $163(30.0)$ & & \\
\hline \multicolumn{7}{|l|}{ Hypertension, $\mathrm{n}(\%)$} \\
\hline No & $59(33.7)$ & $34(15.5)$ & $19(12.8)$ & $112(20.6)$ & \multirow[t]{2}{*}{27.388} & \multirow[t]{2}{*}{$<0.001$} \\
\hline Yes & $116(66.3)$ & $186(84.5)$ & $129(87.2)$ & $431(79.4)$ & & \\
\hline \multicolumn{7}{|l|}{ Hyperlipidemia, n (\%) } \\
\hline No & $104(59.4)$ & $88(40.2)$ & $75(50.7)$ & $267(49.3)$ & \multirow[t]{2}{*}{14.578} & \multirow[t]{2}{*}{0.001} \\
\hline Yes & $71(40.6)$ & $131(59.8)$ & $73(49.3)$ & $275(50.7)$ & & \\
\hline \multicolumn{7}{|l|}{ Smoking, n (\%) } \\
\hline No & $128(73.1)$ & $119(54.1)$ & $90(60.8)$ & $337(62.1)$ & \multirow[t]{2}{*}{15.161} & \multirow[t]{2}{*}{0.001} \\
\hline Yes & $47(26.9)$ & $101(45.9)$ & $58(39.2)$ & $206(37.9)$ & & \\
\hline \multicolumn{7}{|l|}{ Wine consumption, n (\%) } \\
\hline No & $167(95.4)$ & $175(79.5)$ & $126(85.1)$ & $468(86.2)$ & \multirow[t]{2}{*}{20.845} & \multirow[t]{2}{*}{$<0.01$} \\
\hline Yes & $8(4.6)$ & $45(20.5)$ & $22(14.9)$ & $75(13.6)$ & & \\
\hline Age, years ${ }^{\mathrm{a}}$ & $61.7 \pm 11.3$ & $65.5 \pm 11.1$ & $62.6 \pm 11.6$ & & 6.2 & 0.002 \\
\hline Systolic blood pressure, $\mathrm{mmHg}^{\mathrm{a}}$ & $140.7 \pm 21.4$ & $152.54 \pm 22.9$ & $157.3 \pm 23.0$ & & 24.1 & $<0.01$ \\
\hline Diastolic blood pressure, $\mathrm{mmHg}^{\mathrm{a}}$ & $79.6 \pm 9.8$ & $85.7 \pm 12.4$ & $88.2 \pm 12.7$ & & 27.5 & $<0.01$ \\
\hline Platelet count $\left(10^{9} / 1\right)^{\mathrm{a}}$ & $233.751 \pm 72.418$ & $244.735 \pm 76.258$ & $247.944 \pm 83.259$ & & 1.559 & 0.211 \\
\hline International normalized ratio ${ }^{a}$ & $1.031 \pm 0.094$ & $1.013 \pm 0.094$ & $1.011 \pm 0.087$ & & 2.097 & 0.124 \\
\hline Plasma fibrinogen $(\mathrm{g} / \mathrm{l})^{\mathrm{a}}$ & $3.368 \pm 0.879$ & $3.984 \pm 1.103$ & $3.659 \pm 0.835$ & & 17.363 & $<0.001$ \\
\hline Total cholesterol $(\mathrm{mmol} / \mathrm{l})^{\mathrm{a}}$ & $4.742 \pm 1.142$ & $5.082 \pm 1.228$ & $4.909 \pm 1.113$ & & 4.039 & 0.018 \\
\hline Triglyceride $(\mathrm{mmol} / \mathrm{l})^{\mathrm{a}}$ & $1.677 \pm 1.236$ & $1.762 \pm 1.071$ & $1.719 \pm 0.972$ & & 0.291 & 0.748 \\
\hline $\mathrm{HDL}(\mathrm{mmol} / \mathrm{l})^{\mathrm{a}}$ & $1.192 \pm 0.407$ & $1.114 \pm 0.566$ & $1.142 \pm 0.463$ & & 1.189 & 0.305 \\
\hline $\mathrm{LDL}(\mathrm{mmol} / \mathrm{l})^{\mathrm{a}}$ & $2.542 \pm 0.907$ & $2.936 \pm 0.997$ & $2.796 \pm 0.984$ & & 7.966 & $<0.001$ \\
\hline $\mathrm{HLR}^{\mathrm{a}}$ & $0.542 \pm 0.357$ & $0.418 \pm 0.212$ & $0.498 \pm 0.454$ & & 8.833 & $<0.001$ \\
\hline Fasting blood glucose $(\mathrm{mmol} / \mathrm{l})^{\mathrm{a}}$ & $5.765 \pm 2.347$ & $7.166 \pm 3.402$ & $6.414 \pm 2.354$ & & 11.659 & $<0.001$ \\
\hline Glycated hemoglobin $(\%)^{\mathrm{a}}$ & $6.243 \pm 1.376$ & $7.219 \pm 2.189$ & $6.613 \pm 1.879$ & & 10.379 & $<0.001$ \\
\hline Homocysteine $(\mu \mathrm{mol} / \mathrm{l})^{\mathrm{a}}$ & $14.301 \pm 4.473$ & $15.051 \pm 6.512$ & $14.640 \pm 6.106$ & & 0.500 & 0.607 \\
\hline
\end{tabular}

${ }^{\mathrm{a}}$ Mean \pm SD. HDL, high-density lipoprotein; LDL, low-density lipoprotein; HLR, HDL/LDL ratio.

effect of hypertension on carotid artery stenosis. Hyperlipidemia was more common among patients with impaired carotid arteries, despite the less evident difference in the incidence $\left(\chi^{2}=10.312 ; \mathrm{P}=0.016\right)$. Notably, other factors, including smoking, alcohol consumption, cholesterol and lipoprotein, were not significantly different between the normal carotid artery and the dysfunctional carotid artery groups. The ages of the patients varied among the groups with different carotid artery lesions. Stenosis occurred more frequently in older patients.

In addition, whether the factors listed in Table I contributed to the development of cerebral artery stenosis was investigated. As shown in Table II, diabetes, hypertension, hyperlipidemia and age were associated with the severity of cerebral artery stenosis, exhibiting a similar pattern to carotid artery atherosclerosis. However, smoking and alcohol consumption was also demonstrated to affect the narrowing of cerebral arteries, contrary to carotid arteries.

Furthermore, whether these factors were involved in the development of neurological deficits, including stroke and progressive stroke, was investigated. The factors that contributed to the severity of cerebral stenosis (Table II) also affected the incidence of stroke (Table III) in a similar manner. This observation also enhances the correlation of stroke and cerebral artery stenosis. However, the data showed that only hyperlipidemia, alcohol consumption and age were significantly different between the patients with or without progressive stroke (Table IV; $\mathrm{P}<0.05$ ).

Multivariate logistic regression analysis. Multiple linear regression analysis revealed that multicollinearity existed 
Table IV. Association between potential risk factors and progressive stroke.

\begin{tabular}{|c|c|c|c|c|c|}
\hline \multirow[b]{2}{*}{ Risk factor } & \multicolumn{2}{|c|}{ Progressive stroke } & \multirow[b]{2}{*}{ Total } & \multirow[b]{2}{*}{ Test statistic } & \multirow[b]{2}{*}{ P-value } \\
\hline & No $(n=368)$ & Yes $(n=60)$ & & & \\
\hline \multicolumn{6}{|l|}{ Gender, n (\%) } \\
\hline Male & $262(71.2)$ & $43(71.7)$ & $305(71.3)$ & 0.006 & 0.940 \\
\hline Female & $106(28.8)$ & $17(28.3)$ & $123(28.7)$ & & \\
\hline \multicolumn{6}{|l|}{ Diabetes, n (\%) } \\
\hline No & $248(67.4)$ & $40(66.7)$ & $288(67.3)$ & 0.012 & 0.912 \\
\hline Yes & $120(32.6)$ & $20(33.3)$ & $140(32.7)$ & & \\
\hline \multicolumn{6}{|l|}{ Hypertension, n (\%) } \\
\hline No & $58(15.8)$ & $13(21.7)$ & $71(16.6)$ & 1.300 & 0.254 \\
\hline Yes & $310(84.2)$ & $47(78.3)$ & $357(83.4)$ & & \\
\hline \multicolumn{6}{|l|}{ Hyperlipidemia, n (\%) } \\
\hline No & $179(48.8)$ & $21(35.0)$ & $200(46.8)$ & 3.929 & 0.047 \\
\hline Yes & $188(51.2)$ & $39(65.0)$ & $227(53.2)$ & & \\
\hline \multicolumn{6}{|l|}{ Smoking, n (\%) } \\
\hline No & $217(59.0)$ & $29(48.3)$ & $246(57.5)$ & 2.387 & 0.122 \\
\hline Yes & $151(41.0)$ & $31(51.7)$ & $182(42.5)$ & & \\
\hline \multicolumn{6}{|l|}{ Wine consumption, n (\%) } \\
\hline No & $318(86.4)$ & $37(61.7)$ & $355(82.9)$ & 22.331 & $<0.01$ \\
\hline Yes & $50(13.6)$ & $23(38.3)$ & $73(17.1)$ & & \\
\hline Age, years ${ }^{\mathrm{a}}$ & $63.5 \pm 11.1$ & $68.8 \pm 12.0$ & & -3.4 & $<0.01$ \\
\hline Systolic blood pressure, $\mathrm{mmHg}^{\mathrm{a}}$ & $153.6 \pm 24.1$ & $152.8 \pm 19.0$ & & 0.3 & 0.766 \\
\hline Diastolic blood pressure, $\mathrm{mmHg}^{\mathrm{a}}$ & $85.7 \pm 12.9$ & $87.1 \pm 11.7$ & & -0.8 & 0.432 \\
\hline Platelet count $\left(10^{9} / 1\right)^{\mathrm{a}}$ & $244.019 \pm 79.526$ & $246.967 \pm 89.006$ & & -0.261 & 0.794 \\
\hline International normalized ratio ${ }^{a}$ & $1.031 \pm 0.118$ & $0.975 \pm 0.099$ & & 3.46 & $<0.01$ \\
\hline Plasma fibrinogen $(\mathrm{g} / \mathrm{l})^{\mathrm{a}}$ & $3.789 \pm 0.982$ & $3.964 \pm 1.135$ & & -1.234 & 0.218 \\
\hline Total cholesterol $(\mathrm{mmol} / \mathrm{l})^{\mathrm{a}}$ & $4.980 \pm 1.229$ & $5.278 \pm 1.148$ & & -1.756 & 0.080 \\
\hline Triglyceride $(\mathrm{mmol} / \mathrm{l})^{\mathrm{a}}$ & $1.706 \pm 1.022$ & $1.686 \pm 0.923$ & & 0.14 & 0.889 \\
\hline $\operatorname{HDL}(\mathrm{mmol} / \mathrm{l})^{\mathrm{a}}$ & $1.121 \pm 0.355$ & $1.204 \pm 0.998$ & & -0.635 & 0.528 \\
\hline $\mathrm{LDL}(\mathrm{mmol} / \mathrm{l})^{\mathrm{a}}$ & $2.840 \pm 1.032$ & $3.032 \pm 0.935$ & & -1.349 & 0.178 \\
\hline $\mathrm{HLR}^{\mathrm{a}}$ & $0.458 \pm 0.298$ & $0.453 \pm 0.459$ & & 0.083 & 0.934 \\
\hline Fasting blood glucose $(\mathrm{mmol} / \mathrm{l})^{\mathrm{a}}$ & $6.610 \pm 2.599$ & $7.782 \pm 4.420$ & & -1.997 & 0.050 \\
\hline Glycated hemoglobin $(\%)^{\mathrm{a}}$ & $7.073 \pm 2.108$ & $6.494 \pm 1.995$ & & 1.917 & 0.056 \\
\hline Homocysteine $(\mu \mathrm{mol} / \mathrm{l})^{\mathrm{a}}$ & $14.847 \pm 6.432$ & $13.731 \pm 5.049$ & & 1.429 & 0.156 \\
\hline
\end{tabular}

${ }^{\mathrm{a}}$ Mean \pm SD. HDL, high-density lipoprotein; LDL, low-density lipoprotein; HLR, HDL/LDL ratio.

between systolic and diastolic pressure, total cholesterol and LDL. Due to extensive variance, two factors (diastolic blood pressure and total cholesterol) were rejected in the model and the results are shown in Table V. The results demonstrated that these factors exhibited significant differences at various levels of carotid artery atherosclerosis (hardened, hardened plaque and stenosis groups), when compared with the normal group. The odds ratio was set at $>1$ for risk factors and otherwise protective factors. As shown in Table VI, the influencing factors of cerebral artery stenosis included age, diabetes, plasma fibrinogen and HLR (HDL/LDL ratio), among which age, diabetes mellitus and plasma fibrinogen were identified as risk factors, whereas HLR was a protective factor. As shown in Table VII, risk factors were also identified for stroke. These included fasting blood glucose and smoking.
However, multivariate analysis of the bivariate correlation between progressive stroke and cerebral artery atherosclerosis exhibited no significant correlation (Table VIII). For effects of fasting blood glucose and plasma fibrinogen (FIB) classification, as shown in Table IX, age, diabetes and smoking were important factors for carotid atherosclerosis. Similarly, age and diabetes were also the important factors in FIB classification of cerebral artery stenosis (Table X).

\section{Discussion}

PCI is a refractory cerebral vascular disease with an incidence rate of $20-30 \%$ in patients with cerebral infarction. PCI often leads to brain deterioration and thereby significantly increases the mortality rate (8-10). The occurrence and development 
Table V. Logistic regression analysis of factors affecting carotid atherosclerosis.

\begin{tabular}{|c|c|c|c|c|c|c|c|}
\hline \multirow[b]{2}{*}{ Risk factor } & \multirow[b]{2}{*}{$\mathrm{B}$} & \multirow[b]{2}{*}{ SE } & \multirow[b]{2}{*}{ Wald } & \multirow[b]{2}{*}{ P-value } & \multirow[b]{2}{*}{ OR value } & \multicolumn{2}{|c|}{$95 \% \mathrm{CI}$ of OR value } \\
\hline & & & & & & Lower limit & Upper limit \\
\hline \multicolumn{8}{|l|}{ Hardening } \\
\hline Intercept & -10.138 & 6.565 & 2.385 & 0.123 & & & \\
\hline Age & 0.063 & 0.030 & 4.445 & 0.035 & 1.066 & 1.004 & 1.130 \\
\hline Systolic blood pressure & 0.029 & 0.016 & 3.045 & 0.081 & 1.029 & 0.996 & 1.063 \\
\hline Platelet count & 0.003 & 0.004 & 0.338 & 0.561 & 1.003 & 0.994 & 1.011 \\
\hline International normalized ratio & 4.015 & 3.747 & 1.148 & 0.284 & 55.413 & 0.036 & $85,759.316$ \\
\hline Plasma fibrinogen & 0.386 & 0.406 & 0.901 & 0.342 & 1.471 & 0.663 & 3.260 \\
\hline Triglyceride & 0.542 & 0.408 & 1.763 & 0.184 & 1.720 & 0.772 & 3.830 \\
\hline $\mathrm{HDL}$ & 1.878 & 0.947 & 3.932 & 0.047 & 6.540 & 1.022 & 41.851 \\
\hline LDL & 0.441 & 0.397 & 1.232 & 0.267 & 1.554 & 0.714 & 3.382 \\
\hline HLR & -0.269 & 1.235 & 0.048 & 0.827 & 0.764 & 0.068 & 8.601 \\
\hline Fasting blood glucose & 0.197 & 0.172 & 1.314 & 0.252 & 1.218 & 0.870 & 1.705 \\
\hline Glycated hemoglobin & -0.929 & 0.363 & 6.546 & 0.011 & 0.395 & 0.194 & 0.805 \\
\hline Homocysteine & 0.037 & 0.065 & 0.314 & 0.576 & 1.037 & 0.912 & 1.179 \\
\hline Gender & -0.319 & 0.781 & 0.167 & 0.683 & 0.727 & 0.157 & 3.356 \\
\hline Diabetes & 2.322 & 1.161 & 3.999 & 0.046 & 10.195 & 1.047 & 99.254 \\
\hline Hypertension & 0.887 & 0.742 & 1.428 & 0.232 & 2.427 & 0.567 & 10.390 \\
\hline Hyperlipidemia & 0.640 & 0.806 & 0.629 & 0.428 & 1.896 & 0.390 & 9.203 \\
\hline Smoking & 1.522 & 0.777 & 3.839 & 0.050 & 4.582 & 1.000 & 21.002 \\
\hline Wine consumption & -0.232 & 0.926 & 0.063 & 0.802 & 0.793 & 0.129 & 4.868 \\
\hline \multicolumn{8}{|l|}{ Plaque formation } \\
\hline Intercept & -13.361 & 6.013 & 4.938 & 0.026 & & & \\
\hline Age & 0.092 & 0.028 & 10.952 & 0.001 & 1.096 & 1.038 & 1.157 \\
\hline Systolic blood pressure & 0.023 & 0.015 & 2.369 & 0.124 & 1.024 & 0.994 & 1.055 \\
\hline Platelet count & 0.004 & 0.004 & 0.785 & 0.376 & 1.004 & 0.996 & 1.012 \\
\hline International normalized ratio & 6.320 & 3.414 & 3.428 & 0.064 & 555.608 & 0.690 & $447,157.189$ \\
\hline Plasma fibrinogen & 0.454 & 0.377 & 1.449 & 0.229 & 1.574 & 0.752 & 3.296 \\
\hline Triglyceride & 0.248 & 0.393 & 0.400 & 0.527 & 1.282 & 0.594 & 2.768 \\
\hline HDL & 1.874 & 0.929 & 4.070 & 0.044 & 6.512 & 1.055 & 40.209 \\
\hline LDL & -0.220 & 0.377 & 0.341 & 0.559 & 0.802 & 0.383 & 1.681 \\
\hline HLR & -1.205 & 1.166 & 1.068 & 0.301 & 0.300 & 0.030 & 2.946 \\
\hline Fasting blood glucose & 0.143 & 0.152 & 0.886 & 0.346 & 1.154 & 0.856 & 1.556 \\
\hline Glycated hemoglobin & -0.367 & 0.288 & 1.628 & 0.202 & 0.693 & 0.394 & 1.218 \\
\hline Homocysteine & 0.056 & 0.061 & 0.844 & 0.358 & 1.058 & 0.938 & 1.193 \\
\hline Gender & 0.092 & 0.702 & 0.017 & 0.896 & 1.096 & 0.277 & 4.341 \\
\hline Diabetes & 2.163 & 1.092 & 3.921 & 0.048 & 8.700 & 1.022 & 77.035 \\
\hline Hypertension & 1.147 & 0.635 & 3.269 & 0.071 & 3.150 & 0.908 & 10.927 \\
\hline Hyperlipidemia & 1.236 & 0.730 & 2.864 & 0.091 & 3.441 & 0.822 & 14.393 \\
\hline Smoking & 1.006 & 0.698 & 2.077 & 0.150 & 2.735 & 0.696 & 10.749 \\
\hline Wine consumption & 0.337 & 0.840 & 0.160 & 0.689 & 01.400 & 0.270 & 7.267 \\
\hline \multicolumn{8}{|l|}{ Stenosis } \\
\hline Intercept & -12.001 & 6.638 & 3.268 & 0.071 & & & \\
\hline Age & 0.104 & 0.033 & 10.273 & 0.001 & 1.110 & 1.041 & 1.183 \\
\hline Systolic blood pressure & 0.016 & 0.016 & 0.898 & 0.343 & 1.016 & 0.983 & 1.049 \\
\hline Platelet count & 0.000 & 0.005 & 0.000 & 0.996 & 1.000 & 0.991 & 1.009 \\
\hline International normalized ratio & 5.469 & 3.692 & 2.194 & 0.139 & 237.189 & 0.171 & $329,261.826$ \\
\hline Plasma fibrinogen & 0.539 & 0.403 & 1.787 & 0.181 & 1.713 & 0.778 & 3.773 \\
\hline Triglyceride & -0.067 & 0.461 & 0.021 & 0.884 & 0.935 & 0.379 & 2.307 \\
\hline HDL & 1.833 & 1.100 & 2.780 & 0.095 & 6.256 & 0.725 & 53.975 \\
\hline LDL & -0.072 & 0.460 & 0.025 & 0.875 & 0.930 & 0.378 & 2.291 \\
\hline HLR & -2.148 & 1.809 & 1.410 & 0.235 & 0.117 & 0.003 & 4.047 \\
\hline Fasting blood glucose & 0.133 & 0.165 & 0.647 & 0.421 & 1.142 & 0.827 & 1.577 \\
\hline Glycated hemoglobin & -0.406 & 0.317 & 1.637 & 0.201 & 0.666 & 0.358 & 1.241 \\
\hline Homocysteine & 0.059 & 0.066 & 0.800 & 0.371 & 1.061 & 0.932 & 1.209 \\
\hline Gender & 0.276 & 0.801 & 0.118 & 0.731 & 1.317 & 0.274 & 6.332 \\
\hline
\end{tabular}


Table V. Continued. Logistic regression analysis of factors affecting the carotid atherosclerosis.

\begin{tabular}{|c|c|c|c|c|c|c|c|}
\hline \multirow[b]{2}{*}{ Risk factor } & \multirow[b]{2}{*}{ B } & \multirow[b]{2}{*}{ SE } & \multirow[b]{2}{*}{ Wald } & \multirow[b]{2}{*}{ P-value } & \multirow[b]{2}{*}{ OR value } & \multicolumn{2}{|c|}{$95 \% \mathrm{CI}$ of OR value } \\
\hline & & & & & & Lower limit & Upper limit \\
\hline Diabetes & 2.237 & 1.151 & 3.778 & 0.052 & 9.362 & 0.982 & 89.303 \\
\hline Hypertension & 1.654 & 0.789 & 4.387 & 0.036 & 5.225 & 1.112 & 24.552 \\
\hline Hyperlipidemia & 0.947 & 0.804 & 1.387 & 0.239 & 2.577 & 0.533 & 12.450 \\
\hline Smoking & 1.449 & 0.767 & 3.570 & 0.059 & 4.259 & 0.947 & 19.142 \\
\hline Wine consumption & 0.161 & 0.929 & 0.030 & 0.862 & 1.175 & 0.190 & 7.251 \\
\hline
\end{tabular}

CI, confidence interval; SE, standard error; OR, odds ratio; HDL, high-density lipoprotein; LDL, low-density lipoprotein; HLR, HDL/LDL ratio.

Table VI. Logistic regression analysis of factors affecting cerebral artery stenosis (significant factors).

\begin{tabular}{|c|c|c|c|c|c|c|c|}
\hline \multirow[b]{2}{*}{ Risk factor } & \multirow[b]{2}{*}{$\mathrm{B}$} & \multirow[b]{2}{*}{ SE } & \multirow[b]{2}{*}{ Wald } & \multirow[b]{2}{*}{ P-value } & \multirow[b]{2}{*}{ OR value } & \multicolumn{2}{|c|}{$95 \%$ CI of OR value } \\
\hline & & & & & & Lower limit & Upper limit \\
\hline Age & 0.030 & 0.012 & 6.174 & 0.013 & 1.031 & 1.006 & 1.056 \\
\hline Diabetes & 1.054 & 0.295 & 12.772 & 0.000 & 2.869 & 1.609 & 5.133 \\
\hline Plasma fibrinogen & 0.299 & 0.147 & 4.148 & 0.042 & 1.348 & 1.011 & 1.797 \\
\hline HLR & -0.925 & 0.420 & 4.841 & 0.028 & 0.396 & 0.174 & 0.904 \\
\hline
\end{tabular}

HLR, high-density lipoprotein/low-density lipoprotein ratio; CI, confidence interval; OR, odds ratio; SE, standard error.

Table VII. Logistic regression analysis of factors affecting stroke.

\begin{tabular}{|c|c|c|c|c|c|c|c|}
\hline \multirow[b]{2}{*}{ Risk factor } & \multirow[b]{2}{*}{$\mathrm{B}$} & \multirow[b]{2}{*}{ SE } & \multirow[b]{2}{*}{ Wald } & \multirow[b]{2}{*}{ P-value } & \multirow[b]{2}{*}{ OR value } & \multicolumn{2}{|c|}{$95 \% \mathrm{CI}$ of OR value } \\
\hline & & & & & & Lower limit & Upper limit \\
\hline \multicolumn{8}{|l|}{ Large artery atherosclerosis } \\
\hline Intercept & -6.995 & 3.664 & 3.645 & 0.056 & & & \\
\hline Age & 0.010 & 0.019 & 0.262 & 0.609 & 1.010 & 0.972 & 1.049 \\
\hline Systolic blood pressure & 0.013 & 0.009 & 1.763 & 0.184 & 1.013 & 0.994 & 1.031 \\
\hline Platelet count & -0.001 & 0.003 & 0.153 & 0.696 & 0.999 & 0.994 & 1.004 \\
\hline International normalized ratio & -1.453 & 2.100 & 0.479 & 0.489 & 0.234 & 0.004 & 14.348 \\
\hline Plasma fibrinogen & 0.356 & 0.235 & 2.294 & 0.130 & 1.427 & 0.901 & 2.261 \\
\hline Triglyceride & -0.048 & 0.208 & 0.053 & 0.817 & 0.953 & 0.635 & 1.432 \\
\hline HDL & 0.021 & 0.750 & 0.001 & 0.978 & 1.021 & 0.235 & 4.441 \\
\hline LDL & 0.381 & 0.411 & 0.858 & 0.354 & 1.464 & 0.654 & 3.279 \\
\hline HLR & -0.690 & 1.890 & 0.133 & 0.715 & 0.502 & 0.012 & 20.399 \\
\hline Fasting blood glucose & 0.400 & 0.174 & 5.302 & 0.021 & 1.492 & 1.061 & 2.097 \\
\hline Glycated hemoglobin & 0.308 & 0.259 & 1.419 & 0.234 & 1.361 & 0.819 & 2.261 \\
\hline Homocysteine & -0.020 & 0.038 & 0.271 & 0.602 & 0.981 & 0.910 & 1.056 \\
\hline Gender & 0.296 & 0.479 & 0.383 & 0.536 & 1.345 & 0.526 & 3.439 \\
\hline Diabetes & 0.111 & 0.552 & 0.040 & 0.841 & 1.117 & 0.379 & 3.294 \\
\hline Hypertension & 0.293 & 0.530 & 0.306 & 0.580 & 1.341 & 0.474 & 3.789 \\
\hline Hyperlipidemia & 0.505 & 0.454 & 1.239 & 0.266 & 1.657 & 0.681 & 4.034 \\
\hline Smoking & 0.183 & 0.464 & 0.155 & 0.694 & 1.200 & 0.483 & 2.983 \\
\hline Wine consumption & 1.635 & 0.728 & 5.041 & 0.025 & 5.127 & 1.231 & 21.358 \\
\hline \multicolumn{8}{|l|}{ Small artery occlusion } \\
\hline Intercept & -5.180 & 3.822 & 1.837 & 0.175 & & & \\
\hline Age & -0.005 & 0.020 & 0.063 & 0.802 & 0.995 & 0.956 & 1.035 \\
\hline Systolic blood pressure & 0.019 & 0.010 & 3.312 & 0.069 & 1.019 & 0.999 & 1.039 \\
\hline Platelet count & 0.003 & 0.003 & 1.292 & 0.256 & 1.003 & 0.998 & 1.008 \\
\hline International normalized ratio & -3.342 & 2.372 & 1.985 & 0.159 & 0.035 & 0.000 & 3.697 \\
\hline
\end{tabular}


Table VII. Continued. Logistic regression analysis of factors affecting stroke.

\begin{tabular}{|c|c|c|c|c|c|c|c|}
\hline \multirow[b]{2}{*}{ Risk factor } & \multirow[b]{2}{*}{ B } & \multirow[b]{2}{*}{$\mathrm{SE}$} & \multirow[b]{2}{*}{ Wald } & \multirow[b]{2}{*}{ P-value } & \multirow[b]{2}{*}{ OR value } & \multicolumn{2}{|c|}{$95 \% \mathrm{CI}$ of OR value } \\
\hline & & & & & & Lower limit & Upper limit \\
\hline Plasma fibrinogen & 0.061 & 0.250 & 0.060 & 0.806 & 1.063 & 0.652 & 1.735 \\
\hline Triglyceride & -0.018 & 0.212 & 0.007 & 0.932 & 0.982 & 0.648 & 1.489 \\
\hline HDL & -1.230 & 0.926 & 1.763 & 0.184 & 0.292 & 0.048 & 1.796 \\
\hline LDL & 0.803 & 0.422 & 3.626 & 0.057 & 2.232 & 0.977 & 5.100 \\
\hline HLR & 2.486 & 1.812 & 1.881 & 0.170 & 12.010 & 0.344 & 418.996 \\
\hline Fasting blood glucose & 0.361 & 0.180 & 4.022 & 0.045 & 1.434 & 1.008 & 2.041 \\
\hline Glycated hemoglobin & 0.196 & 0.271 & 0.523 & 0.469 & 1.217 & 0.715 & 2.069 \\
\hline Homocysteine & -0.054 & 0.042 & 1.671 & 0.196 & 0.947 & 0.873 & 1.028 \\
\hline Gender & 0.285 & 0.510 & 0.312 & 0.577 & 1.330 & 0.489 & 3.615 \\
\hline Diabetes & 0.005 & 0.607 & 0.000 & 0.994 & 1.005 & 0.306 & 3.302 \\
\hline Hypertension & 0.736 & 0.593 & 1.542 & 0.214 & 2.088 & 0.653 & 6.679 \\
\hline Hyperlipidemia & -0.128 & 0.495 & 0.067 & 0.796 & 0.880 & 0.333 & 2.322 \\
\hline Hyperlipidemia & 0.068 & 0.501 & 0.018 & 0.892 & 1.070 & 0.401 & 2.855 \\
\hline Smoking & 1.513 & 0.768 & 3.886 & 0.049 & 4.542 & 1.009 & 20.455 \\
\hline
\end{tabular}

CI, confidence interval; OR, odds ratio; SE, standard error; HDL, high-density lipoprotein; LDL, low-density lipoprotein; HLR, HDL/LDL ratio.

Table VIII. Logistic regression analysis of factors affecting progressive stroke.

\begin{tabular}{|c|c|c|c|c|c|c|c|}
\hline \multirow[b]{2}{*}{ Risk factor } & \multirow[b]{2}{*}{$\mathrm{B}$} & \multirow[b]{2}{*}{ SE } & \multirow[b]{2}{*}{ Wald } & \multirow[b]{2}{*}{ P-value } & \multirow[b]{2}{*}{ OR value } & \multicolumn{2}{|c|}{$95 \% \mathrm{CI}$ of OR value } \\
\hline & & & & & & Lower limit & Upper limit \\
\hline Age & 0.067 & 0.018 & 14.401 & $<0.001$ & 1.070 & 1.033 & 1.108 \\
\hline Wine consumption & 1.724 & 0.400 & 18.570 & 0.001 & 5.608 & 2.560 & 12.286 \\
\hline International normalized ratio & -6.955 & 2.292 & 9.203 & 0.002 & 0.001 & 0.000 & 0.085 \\
\hline Fasting blood glucose & 0.314 & 0.079 & 15.916 & $<0.001$ & 1.369 & 1.173 & 1.598 \\
\hline Glycated hemoglobin & -0.553 & 0.158 & 12.290 & $<0.001$ & 0.575 & 0.423 & 0.784 \\
\hline
\end{tabular}

Table IX. Effect of fasting blood glucose and FIB classification on carotid atherosclerosis.

\begin{tabular}{|c|c|c|c|c|c|c|c|}
\hline \multirow[b]{2}{*}{ Risk factor } & \multirow[b]{2}{*}{$\mathrm{B}$} & \multirow[b]{2}{*}{ SE } & \multirow[b]{2}{*}{ Wald } & \multirow[b]{2}{*}{ P-value } & \multirow[b]{2}{*}{ OR value } & \multicolumn{2}{|c|}{$95 \% \mathrm{CI}$ of OR value } \\
\hline & & & & & & Lower limit & Upper limit \\
\hline \multicolumn{8}{|l|}{ Hardening } \\
\hline Intercept & -13.523 & 6.101 & 4.912 & 0.027 & & & \\
\hline Age & 0.066 & 0.031 & 4.533 & 0.033 & 1.069 & 1.005 & 1.136 \\
\hline Systolic blood pressure & 0.028 & 0.017 & 2.821 & 0.093 & 1.029 & 0.995 & 1.063 \\
\hline Platelet count & 0.002 & 0.004 & 0.236 & 0.627 & 1.002 & 0.993 & 1.011 \\
\hline International normalized ratio & 4.318 & 3.937 & 1.202 & 0.273 & 75.015 & 0.033 & $168,551.521$ \\
\hline Triglyceride & 0.732 & 0.445 & 2.708 & 0.100 & 2.079 & 0.870 & 4.971 \\
\hline HDL & 1.812 & 1.011 & 3.215 & 0.073 & 6.124 & 0.845 & 44.394 \\
\hline LDL & 0.551 & 0.413 & 1.780 & 0.182 & 1.735 & 0.772 & 3.898 \\
\hline HLR & -0.175 & 1.264 & 0.019 & 0.890 & 0.839 & 0.070 & 10.005 \\
\hline Glycated hemoglobin & -0.872 & 0.345 & 6.381 & 0.012 & 0.418 & 0.212 & 0.822 \\
\hline Homocysteine & 0.025 & 0.065 & 0.150 & 0.698 & 1.026 & 0.902 & 1.166 \\
\hline Diabetes & 2.883 & 1.210 & 5.681 & 0.017 & 17.875 & 1.669 & 191.421 \\
\hline Hypertension & 0.906 & 0.801 & 1.280 & 0.258 & 2.474 & 0.515 & 11.888 \\
\hline Hyperlipidemia & 0.283 & 0.835 & 0.115 & 0.735 & 1.327 & 0.258 & 6.816 \\
\hline
\end{tabular}


Table IX. Continued. Effect of fasting blood glucose and FIB classification on carotid atherosclerosis.

\begin{tabular}{|c|c|c|c|c|c|c|c|}
\hline \multirow[b]{2}{*}{ Risk factor } & \multirow[b]{2}{*}{$\mathrm{B}$} & \multirow[b]{2}{*}{ SE } & \multirow[b]{2}{*}{ Wald } & \multirow[b]{2}{*}{ P-value } & \multirow[b]{2}{*}{ OR value } & \multicolumn{2}{|c|}{$95 \% \mathrm{CI}$ of OR value } \\
\hline & & & & & & Lower limit & Upper limit \\
\hline Smoking & 1.578 & 0.796 & 3.934 & 0.047 & 4.845 & 1.019 & 23.043 \\
\hline Wine consumption & -0.026 & 0.989 & 0.001 & 0.979 & 0.974 & 0.140 & 6.761 \\
\hline Gender & -0.274 & 0.816 & 0.113 & 0.737 & 0.760 & 0.154 & 3.760 \\
\hline Fasting blood glucose 1 & 0.313 & 1.013 & 0.096 & 0.757 & 1.368 & 0.188 & 9.965 \\
\hline Fasting blood glucose 2 & 0.133 & 1.014 & 0.017 & 0.896 & 1.142 & 0.157 & 8.328 \\
\hline Fasting blood glucose 3 & -0.260 & 0.992 & 0.069 & 0.793 & 0.771 & 0.110 & 5.388 \\
\hline Plasma fibrinogen 1 & -1.408 & 1.031 & 1.865 & 0.172 & 0.245 & 0.032 & 1.846 \\
\hline Plasma fibrinogen 2 & 0.242 & 0.978 & 0.061 & 0.805 & 1.273 & 0.187 & 8.654 \\
\hline Plasma fibrinogen 3 & -0.125 & 0.936 & 0.018 & 0.893 & 0.882 & 0.141 & 5.523 \\
\hline \multicolumn{8}{|l|}{ Plaque formation } \\
\hline Intercept & -17.606 & 5.621 & 9.811 & 0.002 & & & \\
\hline Age & 0.094 & 0.029 & 10.782 & 0.001 & 1.099 & 1.039 & 1.162 \\
\hline Systolic blood pressure & 0.023 & 0.016 & 2.254 & 0.133 & 1.024 & 0.993 & 1.055 \\
\hline Platelet count & 0.004 & 0.004 & 0.795 & 0.373 & 1.004 & 0.996 & 1.012 \\
\hline International normalized ratio & 6.246 & 3.601 & 3.009 & 0.083 & 516.125 & 0.444 & $599,451.170$ \\
\hline Triglyceride & 0.358 & 0.423 & 0.717 & 0.397 & 1.431 & 0.624 & 3.280 \\
\hline HDL & 1.847 & 0.989 & 3.490 & 0.062 & 6.343 & 0.913 & 44.055 \\
\hline LDL & -0.133 & 0.394 & 0.113 & 0.736 & 0.876 & 0.405 & 1.896 \\
\hline HLR & -1.207 & 1.202 & 1.010 & 0.315 & 0.299 & 0.028 & 3.151 \\
\hline Glycated hemoglobin & -0.275 & 0.259 & 1.122 & 0.289 & 0.760 & 0.457 & 1.263 \\
\hline Homocysteine & 0.054 & 0.061 & 0.769 & 0.381 & 1.055 & 0.936 & 1.190 \\
\hline Diabetes & 2.713 & 1.146 & 5.609 & 0.018 & 15.076 & 1.596 & 142.377 \\
\hline Hypertension & 1.384 & 0.692 & 3.998 & 0.046 & 3.991 & 1.028 & 15.500 \\
\hline Hyperlipidemia & 0.877 & 0.753 & 1.355 & 0.244 & 2.403 & 0.549 & 10.518 \\
\hline Smoking & 1.105 & 0.719 & 2.364 & 0.124 & 3.019 & 0.738 & 12.350 \\
\hline Wine consumption & 0.727 & 0.892 & 0.664 & 0.415 & 2.069 & 0.360 & 11.890 \\
\hline Gender & 0.033 & 0.737 & 0.002 & 0.964 & 1.034 & 0.244 & 4.385 \\
\hline Fasting blood glucose 1 & 0.582 & 0.915 & 0.404 & 0.525 & 1.790 & 0.298 & 10.766 \\
\hline Fasting blood glucose 2 & 0.422 & 0.916 & 0.212 & 0.645 & 1.525 & 0.253 & 9.172 \\
\hline Fasting blood glucose 3 & -0.182 & 0.904 & 0.040 & 0.841 & 0.834 & 0.142 & 4.902 \\
\hline Plasma fibrinogen 1 & -1.163 & 0.929 & 1.568 & 0.211 & 0.313 & 0.051 & 1.930 \\
\hline Plasma fibrinogen 2 & 0.262 & 0.893 & 0.086 & 0.769 & 1.300 & 0.226 & 7.476 \\
\hline Plasma fibrinogen 3 & -0.938 & 0.879 & 1.137 & 0.286 & 0.392 & 0.070 & 2.194 \\
\hline \multicolumn{8}{|l|}{ Stenosis } \\
\hline Intercept & -17.095 & 6.254 & 7.471 & 0.006 & & & \\
\hline Age & 0.094 & 0.033 & 7.945 & 0.005 & 1.099 & 1.029 & 1.173 \\
\hline Systolic blood pressure & 0.013 & 0.017 & 0.603 & 0.437 & 1.013 & 0.980 & 1.048 \\
\hline Platelet count & -0.001 & 0.005 & 0.041 & 0.839 & 0.999 & 0.990 & 1.008 \\
\hline International normalized ratio & 6.102 & 3.870 & 2.487 & 0.115 & 446.693 & 0.227 & $878,531.134$ \\
\hline Triglyceride & -0.010 & 0.480 & 0.000 & 0.984 & 0.990 & 0.387 & 2.535 \\
\hline HDL & 1.555 & 1.182 & 1.729 & 0.189 & 4.733 & 0.466 & 48.022 \\
\hline LDL & -0.034 & 0.492 & 0.005 & 0.945 & 0.966 & 0.368 & 2.536 \\
\hline HLR & -1.891 & 1.908 & 0.982 & 0.322 & 0.151 & 0.004 & 6.353 \\
\hline Glycated hemoglobin & -0.157 & 0.288 & 0.295 & 0.587 & 0.855 & 0.486 & 1.504 \\
\hline Homocysteine & 0.060 & 0.067 & 0.799 & 0.371 & 1.062 & 0.931 & 1.210 \\
\hline Diabetes & 2.665 & 1.215 & 4.808 & 0.028 & 14.367 & 1.327 & 155.573 \\
\hline Hypertension & 1.943 & 0.861 & 5.091 & 0.024 & 6.979 & 1.291 & 37.737 \\
\hline Hyperlipidemia & 0.685 & 0.827 & 0.686 & 0.407 & 1.985 & 0.392 & 10.048 \\
\hline Smoking & 1.584 & 0.787 & 4.055 & 0.044 & 4.874 & 1.043 & 22.773 \\
\hline Wine consumption & 0.555 & 0.974 & 0.326 & 0.568 & 1.743 & 0.259 & 11.746 \\
\hline Gender & 0.161 & 0.846 & 0.036 & 0.849 & 1.174 & 0.224 & 6.161 \\
\hline Fasting blood glucose 1 & 1.193 & 1.063 & 1.260 & 0.262 & 3.298 & 0.411 & 26.501 \\
\hline Fasting blood glucose 2 & 0.979 & 1.089 & 0.808 & 0.369 & 2.663 & 0.315 & 22.524 \\
\hline Fasting blood glucose 3 & 1.366 & 1.009 & 1.835 & 0.176 & 3.921 & 0.543 & 28.311 \\
\hline Plasma fibrinogen 1 & -1.024 & 0.994 & 1.061 & 0.303 & 0.359 & 0.051 & 2.521 \\
\hline
\end{tabular}


Table IX. Continued. Effect of fasting blood glucose and FIB classification on carotid atherosclerosis.

\begin{tabular}{|c|c|c|c|c|c|c|c|}
\hline \multirow[b]{2}{*}{ Risk factor } & \multirow[b]{2}{*}{ B } & \multirow[b]{2}{*}{ SE } & \multirow[b]{2}{*}{ Wald } & \multirow[b]{2}{*}{ P-value } & \multirow[b]{2}{*}{ OR value } & \multicolumn{2}{|c|}{$95 \%$ CI of OR value } \\
\hline & & & & & & Lower limit & Upper limit \\
\hline Plasma fibrinogen 2 & -1.151 & 1.053 & 1.194 & 0.275 & 0.316 & 0.040 & 2.492 \\
\hline Plasma fibrinogen 3 & -0.871 & 0.936 & 0.866 & 0.352 & 0.418 & 0.067 & 2.620 \\
\hline
\end{tabular}

FIB, fibrinogen; CI, confidence interval; OR, odds ratio; SE, standard error; HDL, high-density lipoprotein; LDL, low-density lipoprotein; HLR, HDL/ LDL ratio.

Table X. Effect of fasting blood glucose and FIB classification on cerebral artery stenosis.

\begin{tabular}{|c|c|c|c|c|c|c|c|}
\hline \multirow[b]{2}{*}{ Factors } & \multirow[b]{2}{*}{$\mathrm{B}$} & \multirow[b]{2}{*}{ SE } & \multirow[b]{2}{*}{ Wald } & \multirow[b]{2}{*}{ P-value } & \multirow[b]{2}{*}{ OR value } & \multicolumn{2}{|c|}{$95 \%$ CI of OR value } \\
\hline & & & & & & Lower limit & Upper limit \\
\hline Gender & 0.033 & 0.361 & 0.009 & 0.926 & 1.034 & 0.509 & 2.100 \\
\hline Age & 0.031 & 0.014 & 4.796 & 0.029 & 1.032 & 1.003 & 1.061 \\
\hline Diabetes & 0.946 & 0.415 & 5.186 & 0.023 & 2.574 & 1.141 & 5.809 \\
\hline Hypertension & 0.185 & 0.404 & 0.210 & 0.647 & 1.203 & 0.545 & 2.659 \\
\hline Hyperlipidemia & 0.585 & 0.352 & 2.759 & 0.097 & 1.794 & 0.900 & 3.576 \\
\hline Smoking & 0.265 & 0.346 & 0.586 & 0.444 & 1.303 & 0.662 & 2.565 \\
\hline Wine consumption & 0.239 & 0.437 & 0.300 & 0.584 & 1.270 & 0.540 & 2.990 \\
\hline Systolic blood pressure & 0.002 & 0.007 & 0.110 & 0.740 & 1.002 & 0.989 & 1.015 \\
\hline Platelet count & 0.000 & 0.002 & 0.035 & 0.852 & 1.000 & 0.997 & 1.004 \\
\hline International normalized ratio & -0.400 & 1.450 & 0.076 & 0.783 & 0.671 & 0.039 & 11.492 \\
\hline Triglyceride & -0.142 & 0.162 & 0.772 & 0.380 & 0.868 & 0.632 & 1.191 \\
\hline HDL & -0.006 & 0.345 & 0.000 & 0.985 & 0.994 & 0.505 & 1.953 \\
\hline LDL & -0.055 & 0.203 & 0.074 & 0.785 & 0.946 & 0.636 & 1.408 \\
\hline HLR & -0.788 & 0.666 & 1.403 & 0.236 & 0.455 & 0.123 & 1.676 \\
\hline Glycated hemoglobin & 0.009 & 0.128 & 0.005 & 0.946 & 1.009 & 0.785 & 1.295 \\
\hline Homocysteine & 0.033 & 0.029 & 1.255 & 0.263 & 1.033 & 0.976 & 1.094 \\
\hline Fasting blood glucose & & & 1.497 & 0.683 & & & \\
\hline Fasting blood glucose 1 & -0.480 & 0.470 & 1.040 & 0.308 & 0.619 & 0.246 & 1.556 \\
\hline Fasting blood glucose 2 & -0.276 & 0.477 & 0.335 & 0.563 & 0.759 & 0.298 & 1.932 \\
\hline Fasting blood glucose 3 & -0.060 & 0.457 & 0.018 & 0.895 & 0.941 & 0.385 & 2.304 \\
\hline Plasma fibrinogen & & & 1.967 & 0.579 & & & \\
\hline Plasma fibrinogen 1 & -0.269 & 0.415 & 0.421 & 0.516 & 0.764 & 0.339 & 1.723 \\
\hline Plasma fibrinogen 2 & -0.427 & 0.402 & 1.129 & 0.288 & 0.653 & 0.297 & 1.434 \\
\hline Plasma fibrinogen 3 & 0.058 & 0.398 & 0.022 & 0.883 & 1.060 & 0.486 & 2.311 \\
\hline
\end{tabular}

HDL, high-density lipoprotein; LDL, low-density lipoprotein; HLR, HDL/LDL ratio; CI, confidence interval; OR, odds ratio; SE, standard error.

of PCI are affected by a number of factors and mechanisms. Among the numerous risk factors, atherosclerosis, stenosis or occlusion of the trunk and main branches of cerebral arteries are major independent risk factors (11). Consistent with a previous study (12), the data of the present study demonstrated that the corresponding vessels in the infarction region had various degrees of vascular sclerosis and stenosis.

Multiple mechanisms of cerebral infarction caused by atherosclerosis have been proposed, including intravascular thrombosis, vascular stenosis and reduced perfusion pressure in terminal cerebral vessels (8). When intravascular plaques detach from the arterial thrombus or atherosclerosis directly involves the perforator vessels, cerebral infarction may occur. The atherosclerotic vessels are more prone to thrombosis, which aggravates the preexisting vascular stenosis or occlusion (13). Thrombosis may exacerbate cerebral ischemia unless collateral circulation is formed in time. When collateral circulation does not form, cerebral infarction becomes progressive. Stenosis of the cerebral vessels is an additional mechanism underlying the progression of infarction (14). The narrowed cerebral vessels are more likely to have local thrombosis. Thrombosis may extend to the distal vessels resulting in stenosis, and detachment of the thrombus from the wall may also cause an arterial embolism $(15,16)$. When stenosis occurs in the internal carotid, vertebral basilar or other medium-sized arteries, blood flow to the distal branches decreases. With low perfusion, the distal narrowed vessels fail to form effective collateral circulation to bypass the blockage. The results of the 
present study revealed that atherosclerotic plaques and stenosis existed in the corresponding vessels of cerebral infarction. These observations indicate that cerebral vascular lesions play an important role in the pathogenesis of PCI. In addition, the current study identified that numerous factors, including age, HDL, glycosylated hemoglobin and blood parameters, correlated with the severity of atherosclerosis, plaque formation and stenosis in the carotid artery. Factors affecting cerebral artery stenosis were also identified, including age, diabetes, plasma fibrinogen and HLR, among which age, diabetes mellitus and plasma fibrinogen were risk factors, while HLR was a protective factor. Therefore, in patients with acute cerebral infarction, early treatment of vascular stenosis and cerebral artery recanalization may improve cerebral perfusion, thus, prevent the progression and recurrence of cerebral infarction. A previous study demonstrated that placing a stent in the narrowed vessels of patients with PCI, particularly in those with artery stenosis when performed within $16 \mathrm{~h}$ of disease onset and treated within $8 \mathrm{~h}$, achieves favorable effects (17). In conclusion, the results of the present study indicate that the lesions of responsible blood vessels play an important role in PCI. The observations provide supporting evidence for interventional therapy for cerebrovascular disease.

\section{Acknowledgements}

This study was supported by the Science and Technology Program for Dongguan Higher Education, Science, Research and Health Care (grant number 201010515000333). The authors would like to thank Forevergen Biosciences for assistance with the experiments and for valuable discussion and 91SCI Company for language editing assistance.

\section{References}

1. Arboix A and Alio J: Acute cardioembolic cerebral infarction: answers to clinical questions. Curr Cardiol Rev 8: 54-67, 2012.

2. Jespersen SN and Østergaard L: The roles of cerebral blood flow, capillary transit time heterogeneity, and oxygen tension in brain oxygenation and metabolism. J Cereb Blood Flow Metab 32: 264-277, 2012
3. Day JS and Adams HP Jr: Delayed catastrophic intracerebral hemorrhage preceded by progressive recovery after carotid stenting for acute ischemic stroke. J Stroke Cerebrovasc Dis 21: 151-154, 2012.

4. Higashida RT, Meyers PM, Connors JJ, et al: Intracranial angioplasty \& stenting for cerebral atherosclerosis: a position statement of the American Society of Interventional and Therapeutic Neuroradiology, Society of Interventional Radiology, and the American Society of Neuroradiology. J Vasc Interv Radiol 16: 1281-1285, 2005.

5. Wenger NK: Prevention of cardiovascular disease in women: highlights for the clinician of the 2011 American Heart Association Guidelines. Adv Chronic Kidney Dis 20: 419-422, 2013.

6. Prasad K, Dash D and Kumar A: Validation of the Hindi version of National Institute of Health Stroke Scale. Neurol India 60: 40-44, 2012.

7. Ferguson GG, Eliasziw M, Barr HW, et al: The North American Symptomatic Carotid Endarterectomy Trial: surgical results in 1415 patients. Stroke 30: 1751-1758, 1999.

8. Sumer M, Ozdemir I and Erturk O: Progression in acute ischemic stroke: frequency, risk factors and prognosis. J Clin Neurosci 10: 177-180, 2003.

9. Østergaard L, Jespersen SN, Mouridsen K, et al: The role of the cerebral capillaries in acute ischemic stroke: the extended penumbra model. J Cereb Blood Flow Metab 33: 635-648, 2013.

10. Saji N, Kimura K, Kawarai T, Shimizu H and Kita Y: Arterial stiffness and progressive neurological deficit in patients with acute deep subcortical infarction. Stroke 43: 3088-3090, 2012.

11. Ellekjaer EF, Wyller TB, Sverre JM and Holmen J: Lifestyle factors and risk of cerebral infarction. Stroke 23: 829-834, 1992.

12. Thanvi B, Treadwell S and Robinson T: Early neurological deterioration in acute ischaemic stroke: predictors, mechanisms and management. Postgrad Med J 84: 412-417, 2008.

13. Hirsh J, Anand SS, Halperin JL, Fuster V; American Heart Association: Guide to anticoagulant therapy: Heparin: a statement for healthcare professionals from the American Heart Association. Circulation 103: 2994-3018, 2001.

14. Castillo J: Deteriorating stroke: diagnostic criteria, predictors, mechanisms and treatment. Cerebrovasc Dis 9 (Suppl 3): 1-8, 1999.

15. Del Bene A, Palumbo V, Lamassa M, Saia V, Piccardi B and Inzitari D: Progressive lacunar stroke: review of mechanisms, prognostic features, and putative treatments. Int J Stroke 7: 321-329, 2012.

16. Roquer J, Rodríguez-Campello A, Gomis M, et al: Acute stroke unit care and early neurological deterioration in ischemic stroke. J Neurol 255: 1012-1017, 2008.

17. Takase K, Murai H, Tasaki R, et al: Initial MRI findings predict progressive lacunar infarction in the territory of the lenticulostriate artery. Eur Neurol 65: 355-360, 2011. 